\title{
Informatisierung der Geographie
}

\section{Einleitung}

Geographie wird heute von den technologischen Veränderungen des Wandels von der Industriegesellschaft zur Informationsgesellschaft mit beeinflußt: Die Telekommunikation ist im Begriff, sich in Kombination mit der Informatik zur Telematik, einer Technologie, zu wandeln, in der Fernsehen und Pressewesen sich mit elektronischer Post in weltweiter Vernetzung (Internet) mit immer leistungsfähigeren Rechnern und entsprechend mächtiger Software zu einem neuen Kommunikationsforum verbinden, das in großen Schritten globale Züge annimmt. Man spricht heute von «Cyberspace» und «Information Highway», und man erwartet im Laufe von wenigen Jahren einen weiteren mächtigen Technologieschub zu sogenannten «Superhighways» mit rund zehnmal größeren Transportkapazitäten für digitale Information (MITCHELL 1995). Virtuelle Räume, virtuelle Marktplätze und die sinkende Bedeutung des physischen Raumes in der Informatikwelt stellen das traditionelle Raumverständnis der Geographie in Frage.

\section{Von der traditionellen Geographie zur Geoinformatik}

Geographie verstehen wir heute als eine Disziplin, die Natur und Gesellschaft mit dem Ziel untersucht, natürliche und soziale Phänomene und Prozesse in ihren gegenseitigen Beziehungen zu erklären - beschreiben, analysieren, interpretieren, bewerten, prognostizieren und planen -, wobei den räumlichen Aspekten besondere Beachtung geschenkt wird (ITTEN 1982). Für unser Thema'sind hier vor allem diejenigen Aspekte der Geographie von Interesse, die informatisierbar bzw. quantifizierbar sind. In der Nachkriegszeit war die Geographie noch weitgehend dominiert durch den landschafts- und länderkundlichen Ansatz, der zwar unterschiedliche Ausrichtungen in sich vereinigte, aber sehr stark von Alfred Hettner geprägt worden war (vgl. SCHULZ 1980). Verallgemeinernde Metaphern dieser Ausrichtung sind das länderkundliche Schema (z.B. HETTNER 1932) und das Geosphärenmodell (z. B. CAROL 1956). Dieses «Kartenschichten»-Konzept ist heute insofern interessant, als auch in der Geoinformatik ein Denken in Informationsebenen anzutreffen ist.

Der Weg' ${ }^{1}$ von der traditionellen, weitgehend qualitativen Geographie zur heutigen Geoinformatik führt über die beiden Teilprozesse der Quantifizierung und der Informatisierung hin zu Geographischen Informationssystemen.

\subsection{Quantifizierung}

Die ersten namhaften Schritte wurden in der sog. «quantitativen Revolution» der Geographie in den 50er Jahren initiiert. Nach einer Attacke des Amerikaners Schaefer (Schaefer 1953) auf R. Hartshorne, den damaligen Doyen des vorherrschenden Ansatzes der Geographie (Hartshorne 1939) erschienen die ersten quantitativen Studien - z.B. Migrationsuntersuchungen durch Hägerstrand in Schweden (Hägerstrand 1957) -, und es bildete sich an der Universität des Staates Washington in Seattle (USA) unter der Leitung von Garrison eine Schule von jungen Doktoranden, die in verschiedenen Gebieten der Humangeographie und Kartographie unter Benutzung von aus der Ökonomie und Psychologie stammenden Methoden quantitative Untersuchungen vorlegten (B. Berry, W. Bunge, M. F. Dacey, A. Getis, D. F. Marble, J. D. Nystuen, W. Tobler u.a.; vgl. JOHNSTON 1991, S. 58-68). Dieser Generation junger Akademiker gelang es im Laufe der frühen sechziger Jahre, in den Vereinigten Staaten trotz Widerständen der traditionellen Geographie zum Teil namhafte Lehrstühle zu besetzen und hier den neuen Ansatz zu verbreiten. Bereits früh erschienen auch Lehrbücher zur neuen Denkweise (Duncan et al. 1961, Bunge 1962). 1965 wurde von der US Academy of Sciences gemeinsam mit dem National Research Council eine Studie zum Thema «The Science of Geography» herausgegeben (NAS/NRC 1965). Entsprechend der allgemeinen Offenheit der sechziger Jahre gegenüber positivistisch-pragmatischem Denken und Machen war die quantitative Revolution so erfolgreich, daß gegen Ende des Jahrzehnts die neue Arbeitsweise weitherum Anerkennung fand. Die «quantitative und theoretische Geographie», wie sich die neue Ausrichtung nannte, hat seit jener Zeit neben verschiedenen anderen Paradigmen ihren anerkannten Platz in der angloamerikanischen, angelsächsischen, nordeuropäischen und etwas später auch in der zentraleuropäischen Geographie.

K. Brassel, Prof. Dr., A. Vckovski, Dr., E. Schmitt, dipl. Geograph, Geographisches Institut der Universität Zürich, Winterthurerstraße 190, 8057 Zürich 
Die ersten Impulse der genannten Bewegung gingen zwar vor allem von Vertretern der Humangeographie aus (Social Physics, Standorttheorie, Regional Science usw.), doch da sich diese quantitativen Verfahren problemlos auf physisch-geographische Phänomene übertragen ließen, wurde die physische Geographie sehr bald mit einbezogen (vgl. z.B. GARRISON und MARBLE 1967), und das neue Paradigma wurde zu so etwas wie einer neuen Klammer zwischen Human- und physischer Geographie. Diese Quantifizierung lief zum Teil synchron zu ähnlichen Tendenzen in Nachbargebieten der physischen Geographie und unterstützte ein näheres Zusammengehen der naturwissenschaftlichen Forschung innerhalb und außerhalb der institutionellen Grenzen der Geographie. Die Quantifizierung war vor allem eine Besonderheit für die Geographie, denn in den reinen Natur- und den Ingenieurwissenschaften hatten entsprechende Techniken schon früher Eingang gefunden.

\subsection{Informatisierung}

Die Informatisierung folgte der Quantifizierung dicht auf dem Fuß, war man doch für die Umsetzung der quantitativen Methoden auf vorerst noch leistungsschwache Computer angewiesen. Der eigentliche Innovationsschub der Informatisierung und seine substantiellen Auswirkungen sind aber etwas später anzusetzen. Vorerst ging es darum, statistische Verfahren und quantitative Techniken in einfache Fortran- oder Algol-Programme umzusetzen und diese mit selektiven Daten experimentell zu erproben. Ferner wurden bereits in den 60 er Jahren erste experimentelle Zeichensysteme für die Kartenproduktion eingesetzt (BICKMORE und BOYLE 1964). Ab 1965 liefen in Kanada erste Studien zu einem nationalen Geographischen Informationssystem (TOMLINSON 1967). Im gleichen Jahr wurde auch an der Harvard University das «Laboratory for Computer Graphics and Spatial Analysis» gegründet, das maßgebliche Entwicklungen hin zu kartographischer Software und zu ersten rasterbasierten Geoinformationssystemen brachte (SCHMIDT und ZAFFT 1975). Ein Entwicklungsschub ermöglichte auch die 1968 gegründete Kommission «Geographic Data Sensing and Processing» der Internationalen Geographenunion (IGU), die nach vierjähriger Arbeit ein erstes Standardwerk für computergestützte Techniken der Raumanalyse präsentierte (TOMLINSON et al. 1972). 1970 wurde in den Vereinigten Staaten der erste computergestützte Zensus durchgeführt, und im gleichen Jahr hat die CIA eine erste globale Umrißdatenbank (World Data Bank I) herausgegeben. 1973 fand in Nottingham ein erster interdisziplinärer Workshop mit dem Titel «Display and Analysis of Spatial Data» (DAVIS und MacCULLAGH 1975) statt, der schon damals Vertreter aus rund 15 verschiedenen Disziplinen vereinigte. Seit diesem Startpunkt beginnt eine immer dichter werdende Sequenz internationaler Konferenzen zur Informatisierung von raumbezogener Information. Nicht zu unterschätzen sind auch die amerikanischen Erdsatellitenprogramme, die ab 1973 eine systematische Erfassung von Informationen der Erdoberfläche ermöglichen (ERTS, Landsat) und damit eine Konvergenz der Bestrebungen von computergestützter Fernerkundung und Raumanalyse einleiten (DAVIS und SIMONETT 1991).

In der zweiten Hälfte der 70er Jahre erscheinen die ersten kommerziellen Softwarepakete, und im Jahre 1978 legt der U.S. Geological Survey mit dem «National Mapping Program» ein Konzept für ein erstes Großprojekt vor, das vorsah, in einem Zeitraum von rund zwölf Jahren die insgesamt 55000 Blätter der amerikanischen Landeskarte $(1: 24000)$ digital aufzubereiten (STARR Und ANDERSON 1991).

Die 80er Jahre bringen namhafte Verbesserungen von kommerziellen Softwarepaketen und Anstrengungen zum Aufbau von nationalen Basisdatenbeständen in verschiedenen Ländern. Weitere Schlüsselereignisse sind der Bericht einer Kommission der britischen Regierung zur Förderung der Fernerkundung und der geographischen Informationsverarbeitung (DEPT. OF THE ENVIRONMENT 1987) sowie der Beschluß der amerikanischen National Science Foundation im Jahre 1988, ein nationales Zentrum zum Studium und zur Förderung der geographischen Informationsanalyse zu gründen (National Center for Geographic Information and Analysis, NCGIA 1989). Ganz generell geht die Entwicklung in den 70er und 80er Jahren von isolierten Programm- und Applikationsentwicklungen zur Integration der räumlichen Informationsverarbeitung in Geographischen Informationssystemen.

\subsection{Geographische Informationssysteme und Geoinformatik}

Geographische Informationssysteme (GIS) sind integrierte Systeme von Hardware, Software und Prozeduren, die die Datenerfassung, Datenverwaltung, Manipulation, Analyse, Modellierung und Darstellung raumkodierter Daten unterstützen und zur Lösung von komplexen Planungs- und Verwaltungsaufgaben dienen (NCGIA 1989). Neben den Hardware- und Softwarekomponenten sind funktionelle GIS auf relevante Daten, Know-how in der räumlichen Informationsanalyse und organisatorisch-administrative Strukturen angewiesen, wobei erfahrungsgemäß die Hauptkostenfaktoren bei der Erfassung und Pflege der Daten liegen. Der Begriff GIS kann dabei lediglich auf die eingesetzte Software, auf die Installation als Ganzes oder aber umfassend auf die eine entsprechende Dienstleistung erbringende Institution bezogen werden. GIS werden heute in sehr vielen Anwendungsbereichen - von der Raumplanung, der Katasterverwaltung, der Verkehrsüberwachung, der Ressourcenbewirtschaftung bis zum Geomarketing eingesetzt; entsprechend der Nutzung sind Typen unterschiedlicher Spezialisierung und unterschiedlicher technischer Charakteristiken (Systemarchitektur, Datenstrukturen usw.) auf dem Markt. Da sich die Anwendungsbereiche thematisch breit auffächern und der In- 
vestitionsbedarf sehr hoch ist, stellt heute die geographische Informationsverarbeitung weltweit ein Milliardenbusine $\beta$ mit beachtlichen Wachstumsraten dar. GIS werden nicht nur in lokalen, regionalen und nationalen Verwaltungen sowie bei internationalen Organisationen eingesetzt, auch die Privatwirtschaft (Banken, Versicherungen, Immobilienhandel usw.) hat in der Zwischenzeit die Geoinformatik entdeckt und nutzt in rapide wachsendem Maße das Analysepotential raumkodierter Information. Aus den üblichen Managementinformationssystemen werden durch Zusatz von Koordinatenattributen (Geokodierung) potente Geoinformationssysteme.

Geoinformatik kann als «die Wissenschaft vom Wesen und der Funktion der Geoinformation (raumbezogene Information), ihrer Verarbeitung in Form von Geodaten sowie der Anwendung von Geoinformationssystemen» (BARTHELME 1995, S.7) verstanden werden. Als Informatikdisziplin widmet sich die Geoinformatik insbesondere auch der technischen Perfektionierung von Verfahren der Datenmodellierung und Datenmanipulation.

Betrachtet man den vielseitigen Einsatz von GIS und Geoinformatik, so ist die «Informatisierung der Geographie» heute weitgehend Realität: Wo immer Anwendungen dies erfordern, werden Objekte geographisch kodiert und räumlichen Analysen zugänglich gemacht. «Geographie» ist so handhabbar und wird von sehr vielen Bereichen des öffentlichen und privaten Lebens real eingesetzt. Der Geographiebegriff wird hier allerdings nicht deckungsgleich zu unserer wissenschaftlichen Disziplin verwendet. Auch ist zu beachten, daß sehr viele geographische Fragestellungen aus grundsätzlichen Überlegungen nicht digital erfaß- und analysierbar sind.

Dessenungeachtet haben Geographie und GIS/Geoinformatik mehrere Gemeinsamkeiten: Die Geodatenverarbeitung hat sich in Anlehnung an die traditionelle geographische Forschung entwickelt, und Geographen haben zur Entwicklung der Geoinformationssysteme namhafte Beiträge geleistet. Mit der Verbesserung der Datenlage und des Potentials der GIS sind sie auch immer wichtigere Hilfsmittel für wissenschaftliche Fragestellungen sowohl in der physischen wie auch in der Humangeographie. GIS stellen unverzichtbare Komponenten in der Ausbildung der Hochschulgeographie dar, und auch im Betrieb solcher Systeme finden Geographinnen und Geographen heute gute Beschäftigungsmöglichkeiten. Stellen wir die Geographie als Disziplin dem Konzept der Geographischen Informationssysteme gegenüber, so lassen sich $z$. B. folgende Analogien erkennen:

a) Sowohl die Geographie als auch GIS - zwar vermehrt auf technischer Ebene - beschäftigen sich mit der Integration von Wissen aus unterschiedlichen Wissensbereichen und sind von ihrem Wesen her interdisziplinär. Die genannte Analogie zwischen dem landschaftskundlichen Sphärenmodell und dem Informationsebenenmodell in Geographischen Informationssystemen illustriert diesen Sachverhalt. b) Geographie und GIS stehen in einem Spannungsfeld zwischen Integration und Spezialisierung: In der Disziplinengeschichte der Geographie zeigen sich immer wieder Sukzessionen und auch die Koexistenz sowohl von integrierenden und spezialisierenden Ansätzen. Aber auch GIS ermöglichen einerseits die Verwaltung von Einzelfakten und die analytische Beantwortung von Detailfragen, können aber - und diese Möglichkeiten werden durch fortschreitende Verbesserungen der Datenlage und der GIS-Werkzeuge laufend erweitert - auch als integrierte Systeme für die Lösung gesamtheitlicher Fragen eingesetzt werden. Die Interaktivität zwischen Mensch und Computer ermöglicht eine sinnvolle Kombination der Synthesefähigkeit des Menschen mit dem Analysepotential des Rechners.

\section{Informatik und Geographie: Trends und Auswirkungen}

Wie oben dargestellt, haben sich die Geoinformatik und ihre Werkzeuge in den letzen Jahrzehnten von einer Spezialdisziplin zu einer in vielen Bereichen eingesetzten Basistechnologie gewandelt. Der Informatisierungsprozeß des Geobereiches ist aber nach wie vor im Gange, und so ist es angebracht, an dieser Stelle über die gegenwärtigen und künftigen Trends und Auswirkungen dieses Prozesses einige Überlegungen anzustellen. Sind es vor allem technologische Entwicklungen, die diese Veränderungen verursachen, so haben sie doch Auswirkungen auf verschiedene Aspekte des gesellschaftlichen Lebens. Unsere Darstellung gliedert sich deshalb in eine Betrachtung von Trends in der technologischen Entwicklung und gesellschaftliche Auswirkungen.

\subsection{Technische Aspekte}

Der Erfolg von Werkzeugen der Geoinformatik in vielen Bereichen führt mehr und mehr dazu, daß sich GIS von spezifisch eingesetzten Systemen zu allgemeinen Standardprodukten entwickeln. Der Aufbau und Unterhalt einer GIS-Infrastruktur soll nicht aufwendiger und teurer sein als andere weit verbreitete Informationstechnologien wie zum Beispiel Textverarbeitungssysteme oder Programme zur Tabellenkalkulation ( GIS für alle»). Von der globalen Vernetzung via Internet erhofft man sich dabei, die Beschaffung geokodierter Informationen zu erleichtern (oder überhaupt zu ermöglichen) und deren Beschaffungs- und Unterhaltskosten - welche heutzutage, wie oben schon erwähnt, einen wesentlichen Teil eines GIS ausmachen - zu reduzieren.

Einfach zu bedienende und zu unterhaltende GIS - sogenannte «Desktop-GIS» - beginnen die großen und teuren Anlagen der 80er Jahre zu ersetzen. Die damit verbundene zusätzliche Verbreitung führt dazu, daß die Einsatzgebiete von GIS größer und für die Hersteller weniger vorhersehbar werden. Als Standardprodukte werden GIS mehr und mehr zu branchenunabhängigen 
Lösungen variabler Funktionalität. Die dadurch erforderte zusätzliche Flexibilität der Systeme hat in der Forschung der Geoinformatik zu neuen, grundsätzlichen Fragestellungen geführt, welche sich unter «Spatial Information Theory» und "Interoperabilität» zusammenfassen lassen. Genauso wie heute verschiedene Textverarbeitungssysteme branchenneutral arbeiten und Texte (wenn auch limitiert) austauschen können, besteht der Wunsch, dasselbe mit verschiedenen GIS bzw. ihren Komponenten zu erreichen. Die Hauptschwierigkeit und deshalb auch große Herausforderung für die Geoinformatik besteht nun darin, daß bei geographischen Informationen die zugrundeliegende Abstraktion der Realität noch weitgehend unsystematisch und sehr disziplinspezifisch ist. Während beispielsweise bei den Textverar- beitungssystemen die verarbeitete Struktur der Informationen (Texte) im allgemeinen recht gut bekannt ist, verwendet man bei geographischen Informationen immer noch verschiedene, teilweise unvereinbare Ansätze zur Modellierung der Realität. Eine systematischere und besser verstandene Theorie geographischer Information wird den Daten- und Komponentenaustauschoüber Disziplin- und Systemgrenzen hinweg erst ermöglichen. Die weltweite Vernetzung durch das Internet erschließt den Benutzern große vorhandene Datenbestände, deren Neuerfassung große Kosten verursachen würde. Wiederverwendbarkeit ist in diesem Zusammenhang einer der wichtigen Motivatoren für Interoperabilität. Einigen der forschungsrelevanten Themen der Interoperabilität ist ein erster Exkurs gewidmet (s. Kasten).

\section{Exkurs I: Interoperabilität und Standardisierung}

GIS werden heute an vielen Orten mit Erfolg eingesetzt. Die allgemeine starke Verbreitung digitaler Hilfsmittel sowie deren Vernetzung haben aber auch die Notwendigkeit größerer Interoperabilität der Systeme gezeigt (VCKOVSKI 1998). Obwohl die Fähigkeit zur Integration unterschiedlicher räumlicher Informationen oft als eine definierende Eigenschaft von GIS bezeichnet wird(MAGUIRE 1991), sind die heute eingesetzten Systeme oft proprietär; sowohl was die verwendeten Prozesse als auch die Datenmodelle angeht. Aus diesem Grunde sind heute viele Aktivitäten in Forschung und Industrie darauf ausgerichtet, die Werkzeuge der Geoinformatik so zu gestalten, daß Daten und Anwendungen mühelos ausgetauscht werden können.

\section{Hindernisse der Daten- und Systemintegration}

In der Praxis bestehen heute immer noch viele Hindernisse, welche bei der Integration von Daten und Systemen (das Ziel der Interoperabilität) überwunden werden müssen:

- Lokalisierung von Daten

- Syntaktische Heterogenitäten

- Semantische Heterogenitäten

- Marktinteressen

Die Lokalisierung von Daten benötigt digitale Bibliotheken und Datenverzeichnisse. Es wurden in den letzten Jahren zwar einige solcher Datenverzeichnisse (manchmal auch Metadatenbanken genannt) aufge- baut, aber meist innerhalb spezifischer Anwendungsgebiete. Es gibt noch kaum disziplin- und anwendungsgebietübergreifende Datenkataloge, welche in einer standardisierten Art und Weise zugreifbar wären. Die gleiche Entwicklung, welche für Druckerzeugnisse durchlaufen worden ist (Standardisierung für bibliographische Informationen, elektronische Kataloge), wird auch für Datenbestände in der Geoinformatik angestrebt.

Sind einmal zu integrierende Daten lokalisiert, dann stellen sich in der Praxis immer noch viele Probleme. Als syntaktische Heterogenitäten werden diejenigen Hindernisse bezeichnet, welche durch unterschiedliche Datenformate, Plattformen, Systemarchitekturen, aber auch Referenzsysteme zustande kommen, durch Transformationen aber oft automatisch überwunden werden können. Syntaktische Heterogenitäten können zu aufwendigen Konversionsprozessen führen, stellen aber nicht ein grundsätzliches Hindernis für die Integration dar.

Semantische Heterogenitäten hingegen können oft nicht direkt überwunden werden, manchmal werden sie sogar nicht einmal erkannt. Diese Heterogenitäten stammen aus unterschiedlichen Sichtweisen, mit welchen Daten erhoben und Systeme entwickelt wurden. Unterschiedliche Sichtweisen in verschiedenen Anwendungsgebieten, die Notwendigkeit, die Realität zu vereinfachen (Modellieren), aber auch Traditionen führen dazu, daß die gleiche Welt mit sehr unterschiedlichen Augen gesehen und unterschiedlich digitalisiert wird. Die Unterschiede reichen von einfachen Differenzen in der Attributierung (was interessiert mich?) bis zu vollständig unterschiedlichen Konzeptionen des Raumes (z. B. me- 


\subsection{Gesellschaftliche Aspekte}

Die neuen Technologien und ihre weitere Verbreitung bringen aber auch Fragestellungen mit sich, die über das rein Technische hinausgehen und in einem weiteren Sinne als gesellschaftliche Problemstellungen betrachtet werden können. Es hat sich deshalb bereits gegen Ende der 80er Jahre unter GIS-Forschern das Bewußtsein breitgemacht, daß neben technischen auch administrative, juristische, sozialwissenschaftliche und weltanschauliche Fragen untersucht werden sollten. So wurden im Forschungsplan des genannten amerikanischen Kompetenzzentrums NCGIA u. a. folgende Fragestellungen aufgenommen (NCGIA 1989, S.132-135):

- Diffusion und Adaptation der GIS-Technologie

- Nutzung und Wertsetzung geographischer Information

- Institutionelle Struktur (und Wandel) von GIS-Einrichtungen

- Legale Aspekte der Geoinformation

- Zugang zu und Verfügbarkeit von geographischer Information

- Datenschutz und Geoinformation
Aber auch das GISDATA-Programm, eine europäische Forschungsinitiative der sozialwissenschaftlichen Abteilung der European Science Foundation (ESF), hat sich mit ähnlichen Fragestellungen auseinandergesetzt (CRAGLIA 1997). Hinzu kommen Untersuchungen, die Anwendungen der Geotechnologie in speziellen Anwendungsgebieten wie dem Gesundheitswesen, der Planung usw. analysieren. Im Rahmen von NCGIA-Forschungsinitiativen wurden Themenbereiche wie «GIS and Society» (HARRIS und WEINER 1996) und «Spatial Technologies, Geographic Information, and the City» (COUCLELIS 1996) untersucht. In diesen Forschungsgruppen sind z.B. folgende Fragestellungen behandelt oder angeregt worden:

- Epistemologie von GIS (z.B. kritische Aspekte des Informationsgehaltes von GIS, historische Aspekte, welche Realitäten vermittelt ein GIS?,Virtualisierung)

- GIS, Dateninstitutionen und Zugang zu Information (z.B. soziale, institutionelle, regionale Privilegien, ethische Fragen, GIS und globaler Wandel, Verletzung der Persönlichkeitssphäre/Datenschutz, Urheberrechtsfragen) trische Räume vs. diskrete Räume). Eine große Gefahr bei semantischen Heterogenitäten besteht darin, daß sie manchmal schwer erkennbar sind. Eine geographische Referenz kann beispielsweise in einem Datensatz als Punkt kodiert sein, obwohl damit nicht nur der Punkt, sondern auch seine Umgebung gemeint ist. Wenn diese Informationen («Mit einem Punkt ist immer auch noch die Umgebung in einem Umkreis von... gemeint») nicht explizit vorhanden sind, ist eine potentielle Inkompatibilität sehr schwer zu erkennen.

Marktinteressen waren in der Vergangenheit ebenfalls ein Hindernis für die Daten- und Systemintegration. Verschiedene Softwarehersteller waren nicht immer interessiert daran, Produkte zu entwerfen, welche mit Konkurrenzprodukten kompatibel sind und zu weniger Herstellerbindung führen. Die Hersteller wurden durch den Mangel an akzeptierten Standards auch kaum dazu verpflichtet. Heute ist aber immer mehr Infrastruktur vorhanden, welche bei neuen Systemen mit berücksichtigt werden muß. Auch sind die Anwender mündiger geworden, so daß viele Hersteller nun ihre Strategie dahingehend geändert haben, daß die Kompatibilität nun sogar ein Verkaufskriterium geworden ist. Datenlieferanten waren hingegen immer an Standardisierungen interessiert, da dies die Verwendbarkeit (und Verkaufbarkeit) von Daten erhöht.

\section{Standardisierung und räumliche Informationstheorie}

Ansätze zur Überwindung dieser Hindernisse bestehen in der Standardisierung. Diese betrifft einerseits eher syntaktische Standardisierung in der Form von Objekt- definitionen und Datenformaten. Auf der anderen Seite wird auch eine semantische Standardisierung oder eigentlich eine «Theorie über räumliche Information» angestrebt.

Die Entwicklung von standardisierten Datenformaten und Schnittstellen, wie sie beispielsweise von der internationalen Standardisierungsorganisation (International Standardisation Organisation, ISO) und dem Open GIS Consortium (OGC, BUEHLER 1994) angestrebt wird, benötigt als Basis eine akzeptierte Vorstellung über die Abstraktion der Realität. Die Abbildung der Realität in eine endliche Menge beschränkter natürlicher Zahlen (die Digitalisierung) erfordert Modelle und Theorien, mit welchen diese Abbildung nachvollzogen und verstanden werden kann. Eine solche Theorie benötigt wiederum eine Formalisierung der Prozesse mit dem Ziel, möglichst viele Zustände mit möglichst wenigen und einfachen Regeln und Symbolen auszudrücken.

Es ist nach wie vor sehr umstritten, ob die Komplexität räumlicher Informationen genügend allgemein formalisiert werden kann, insbesondere wenn auch kognitive und kulturelle Aspekte mit einbezogen werden. Trotzdem ist es ein fruchtbarer Forschungsansatz, diese Formalisierung zu versuchen. Auch wenn dabei nicht eine allgemeingültige Theorie räumlicher Informationen aufgestellt werden kann, sind doch ein besseres Verständnis dieser Informationsart und eine bessere Basis für Standardisierungsanstrengungen als wünschbare Nebenprodukte dieser Forschung zu werten. 
- Gesellschaftliche Auswirkungen des technologischen Wandels (Enträumlichung des Raums - Raum ohne Ausdehnung, Beschleunigung wirtschaftlicher und sozialer Prozesse, Entstehung einer Informationsklassengesellschaft, Einflüsse auf die Rollen der Geschlechter, Informationsüberflutung, Änderung des Produzenten-Konsumenten-Verhältnisses, neue Organisationsstrukturen, z. B. virtuelle Unternehmen)

- Alternative GIS-Modelle (z.B. föderalistisch statt zentralistisch, individuelle Freiheit/Entfaltungsmöglichkeiten vs. Kontrolle, Auswirkungen von unsicherer Information in GIS, virtuelle Realitäten, Auswirkungen der Internettechnologie)

- GIS und lokale Gemeinschaften (lokales Wissen, Einfluß der neuen Technologie auf lokale Strukturen, GIS für partizipative Gemeindemodelle)
- Räumliche Technologien, GIS und städtischer Raum (Zugriff auf Technologie und räumliche Interaktion, Verkehrsverhalten, Investitionsverhalten usw.)

Diese Fragestellungen gehen über die traditionell aktive Gruppierung von GIS-Forschern hinaus und werden von Juristen, Informationswissenschaftern, Ökonomen und anderen mit bearbeitet. Auf der anderen Seite haben sich mit diesen Themen auch innerhalb der Geographie neue Synergien zwischen GIS-Spezialisten und Wirtschafts- und Sozialgeographen ergeben.

Aus dem Katalog dieser Themenbereiche soll in einem zweiten Exkurs als exemplarisches Beispiel die sog. Virtualisierung, die Substitution der realen Welt durch virtuelle Darstellungen im Rahmen der geographischen Informationsgesellschaft, diskutiert werden (s. Kasten).

\section{Exkurs II: Virtualisierung und Abbildung}

Die Anstrengungen im Bereich der Geoinformatik, die Vernetzung und Interoperabilität isolierter Systeme voranzutreiben, stehen ganz in der Tradition der Geographie, die Welt abzubilden. Geographische Informationssysteme lösen vermehrt die Karte ab und dienen wie diese als Instrument, die Welt zu überschauen, zu beschreiben und den planvollen Zugriff zu ermöglichen. Die «Verdopplung» der aktualen (Außen-)Welt in eine virtuelle ${ }^{2}$ (informationell geklonte), vernetzte und interoperable Daten(innen)welt scheint folgerichtig im Sinn einer Annäherung an die Realität. Zum einen verlangt das Denken in globaler Dimension nach weltweiten und immer detailreicheren Daten, aber auch die Koordination der Aktivitäten einzelner Ämter (Gas-, Wasser-, Stromversorgung, Raumplanung, Umweltschutz usw.) spricht für die Vernetzung der Systeme. Zum anderen bietet sich heute neben der Bereitstellung flächendeckender Daten grundsätzlich die Möglichkeit an, auf den Informations flu $\beta$ selbst zuzugreifen; soließen sich beispielsweise (durch hochfrequente Standortbestimmung mittels Global Positioning Systems erfaßte) Verkehrsflüsse in Echtzeit überwachen und steuern.

\section{Vernetzung, Interoperabilität und Standards}

Voraussetzung für Vernetzung und Interoperabilität ist die Einhaltung gewisser Standards. Die Standardisierung der Datenformate erlaubt es, einen Datensatz A mit einem Datensatz B in Beziehung zu bringen, ohne $\mathrm{da} \beta$ dabei der Hauptaufwand in die Datenintegration investiert werden muß (was Ressourcen für die eigentliche Analyse freisetzt). Mit sogenannten Metadaten (von Angaben über Erfassungsmethode, Maßstab und Genauigkeit bis zur Einschreibung der optimalen
Interpolationsmethode) angereicherte Daten können durch automatisch ablaufende Prozeduren (vor)verarbeitet werden. Und mit zunehmender Standardisierung können solche Automatismen ausgebaut werden, was für eine effiziente Datenverarbeitung wünschenswert ist (v. a. bei dem zu erwartenden Datenvolumen). Anders ausgedrückt, beruht die Effizienz des Standards auf seiner Unsichtbarkeit: Die in den Standard investierte Reflexionsleistung muß nicht weiter oder wiederholt reflektiert werden, was darauf aufbauende Arbeit erleichtert oder erst möglich macht. Der Standard ist Hintergrund bzw. Untergrund. Und es sind die standardisierten Daten, die über standardisierte Kanäle zirkulieren, die nun das «vorgefundene» Ausgangsmaterial bilden.

\section{Virtualität und Realität}

Information und Wissen wird weltweit zugreifbar und von der Bindung an Einzelne befreit, und somit eröffnet sich auf der dadurch entstehenden «Wissensoberfläche» ein ungeahnter Freiraum. Das bisherige Wissen Einzelner, nunmehr Artefakt und überindividuelles Bauteil, wird nun Wissen über den Umgang mit Wissen. DieErfahrungswelt, worin Wissen entstehen kann, verlagert sich: Der Bezugspunkt und somit die neue Welt der Gegenstände ist das im Netz repräsentierte, gesammelte globale Faktenwissen. Die virtuelle Welt wird in dem Maß zur wirkungsvollen, eigengesetzlichen Realität, als die «darunterliegende» Wirklichkeit in Standardisierungen verschwindet.

Damit verhilft der Versuch, die Welt in einer virtuellen abzubilden, nicht zu einem besseren Überblick und Verständnis der Realität, sondern führt vielmehr zu einer Neudefinition der Wirklichkeit. Diese Einsicht ist nicht neu, wird aber durch die Informatisierung offensichtlicher und weit schwieriger zu übersehen. 


\section{Schlußbetrachtungen}

Wir haben vorerst den Werdegang von der traditionellen Geographie über eine Quantifizierung und die Informatisierung zu den heute weit über die Geographie hinaus eingesetzten Geographischen Informationssystemen skizziert und dabei gesehen, daß diese technologische Richtung der Geographie schon sehr früh in einem interdisziplinären Umfeld agiert hat. Neben Geologen, Hydrologen, Kartographen, Planern haben bei der «Informatisierung der Geographie» technische Fachwissenschafter wie Informatiker, Geodäten, Mathematiker und Statistiker mitgewirkt, wobei die Geographen durchaus namhafte Beiträge geleistet haben.

Kann man die Periode von 1960 bis ca. 1975 als Pionierphase mit ersten Automatisierungsversuchen und Softwareentwicklungen an den Hochschulen betrachten, so standen in der Phase zwischen 1975 und 1990 die GIS-Implementation bei ersten großen Datenanbietern und die Entwicklung erster Generationen von kommerzieller GIS-Software im Vordergrund. Die 90er Jahre bringen dann eine immense Ausweitung der GIS-Nutzung, die Vernetzung von administrativen Systemen und die Herausbildung eines kommerziellen GIS-Dienstleistungssektors, und ab 1995 wird der Ausweitungsund Integrationsproze $ß$ weiter beschleunigt durch die Ausbreitung der Internettechnologie.

Mit der Perfektionierung der technischen Komponenten hat unter den Geographinnen und Geographen im GISBereich eine eigentliche Rückbesinnung auf ihre Kernaufgaben stattgefunden. So wird die Entwicklung effizienter Algorithmen vermehrt den Informatikern, die Technik der Datenhaltung vermehrt den Geodäten, die Entwicklung von Kartierprogrammen vermehrt den Kartographen überlassen und die räumliche Analyse mit neuen Mitteln als zentrale Aufgabe betrachtet. Dies äußert sich einerseits darin, daß sowohl in der Humanwie auch in der physischen Geographie GIS zu alltäglichen Werkzeugen der Forschung geworden sind. Andererseits stehen auch bei methodisch interessierten $\mathrm{Geo}$ graphen nicht mehr die GI-Systeme im Vordergrund; vielmehr wird das Akronym GIS vermehrt zur Bezeichnung von "Geographic Information Science» verwendet.

Geographic Information Science wird als grundlegendes Forschungsgebiet betrachtet, das geographische Konzepte und ihre Nutzung im Kontext geographischer Information des Informationszeitalters neu definiert. Es untersucht die fundamentalen Themen traditioneller raumbezogener Fachgebiete und bezieht dabei auch neuere Erkenntnisse der kognitiven und Informationswissenschaften mit ein (GOODCHILD 1992, NCGIA 1995). Ziele sind nicht die Technologie selbst, sondern raumbezogene Erkenntnisse, die mit Hilfe der Technologie gewonnen werden mögen, aber auch Fragen, inwiefern solche Technologien notwendig oder hinreichend sind. Spezielle Themen der geographischen Informationswissenschaft sind z.B. die Konzeptualisierung der geographischen Welt, die Erfassung und Darstellung geo- graphischer Realität unter Berücksichtigung von Konzepten wie Zuverlässigkeit und Genauigkeit, ihre Speicherung, Transformation, Analyse und Visualisierung, ihr Einsatz in Entscheidungsprozessen und nicht zuletzt gesellschaftsrelevante Fragestellungen. In ihrem Rahmen sind - im interdisziplinären Konzert mit den technischen Informationswissenschaften - sowohl Computerspezialisten, quantitative Modellierer wie auch Geographinnen und Geographen anderer Ausrichtung gefordert, Beiträge zur Lösung von Fragen des Informationszeitalters zu leisten.

\section{Anmerkungen}

1 Der geschichtliche Abriß ist hier stark vereinfacht. Detailliertere Darstellungen finden sich in JOHNSTON 1991, S. 51-135, bzw. COPPOCK und RHIND 1991.

2 virtuell (mlat. virtualis, zu lat. virtus = Tüchtigkeit; Mannhaftigkeit, zu: vir, viril): entsprechend seiner Anlage als Möglichkeit vorhanden, die Möglichkeit zu etwas in sich begreifend (Duden, deutsches Universalwörterbuch 1989).

\section{Literatur}

BARTHELME, N. (1995): Geoinformatik - Modelle, Strukturen, Funktionen, Berlin: Springer 1995

BICKMORE, D. P., and BOYLE, A. R. (1964): An Automated System of Cartography, Techn. Symposium der Int. Cartogr. Association, Edinburgh

BUEHLER, K. (1994): OGIS Geodata Model Overview, OGIS project documentation 94-019, Maryland: The Open GIS Consortium (http://www.opengis.org)

BUNGE, W. (1962): Theoretical Geography, Lund Studies in Geography, Series C, Vol. 1, Lund: Gleerup

CAROL, H. (1956): Zur Diskussion um Landschaft und Geographie, Geographica Helvetica, Jg. 9, S. 111-113

COPPOCK, J.T., and RHIND, D.W. (1991): The History of GIS, in: Maguire, D. J., Goodchild, M. F., Rhind, D. W., (eds.) Geographical Information Systems: principles and applications, London: Longman, Vol. 1., pp. 21-43

CRAGLIA, M. (ed.) (1997): Geographic Information Research at the Millenium: GISDATA Final Conference, Le Bischenberg (Fr), Strassburg: ESF

COUCLELIS, H. (1996): Spatial Technologies, Geographic Information, and the City, Technical Report 96-10, National Center for Geographic Information and Analysis, Santa Barbara, $220 \mathrm{pp}$.

DAVIS, F.W., and SIMONETT, D. S. (1991): GIS and Remote Sensing. In: Maguire, D. J., Goodchild, M. F., Rhind, D. W., (eds.) Geographical Information Systems: principles and application,. London: Longman, Vol. 2, pp. 191-217

DAVIS, J.C., and MacCULLAGH, M.C. (1975): Display and Analysis of Spatial Data, London: Wiley 
DEPARTMENT OF THE ENVIRONMENT (1987): Handling Geographic Information, Report of the Committee of Enquiry, London: her Majesty's Stationery Office, 208 pp.

DUNCAN, O.D., CUZZORT, R. P., and DUNCAN, B. (1961): Statistical Geography: Problems in Analyzing Areal Data, Illinois: The Free Press of Glencoe, $191 \mathrm{pp}$.

GARRISON, W. L., and MARBLE, D. F. (1967): Quantitative Geography. Part I: economic and cultural topics; Part II: physical and cartographic topics, Northwestern University Studies in Geography, Vol. 13 and 14, Evanston, Illinois GOODCHILD, M. F. (1992): Geographic Information Science, Intern. Journal of GIS, Vol. 5., No. 1, pp. 31-46

HÄGERSTRAND, T.; (1957): Migration and Area: Survey of a Sample of Swedish Migration Fields and Hypothetical Considerations on their Genesis. In: Hannerberg, D., Hägerstrand, T., and Odeving, B.: Migration in Sweden: A Symposium. Lund Studies in Geography, Series B, No. 13 HARRIS, T., and WEINER, D. (1996): GIS and Society: The Social Implications of How People, Space, and Environment are Represented in GIS, Technical Report 96-10, National Center for Geographic Information and Analysis, Santa Barbara, $220 \mathrm{pp}$.

HARTSHORNE, R. (1939): The Nature of Geography, Westport, Conn.: Greenwood Press (Reprint 1977)

HEITNER, A. (1932): Das Länderkundliche Schema, Geographischer Anzeiger, Jg., 33, S. 1-6

ITTEN, K. (1982): Beitrag zu einer Neudefinition der Geographie, Geographica Helvetica, 37. Jg., Nr. 1, S. 35-37

JOHNSTON, R.J. (1991): Geography and Geographers: Anglo-American Human Geography since 1945, London, New York: Arnold

MAGUIRE, D. J. (1991): An overview and definition of GIS. In: Maguire, D. J., Goodchild, M. F., Rhind, D. W., (eds.) Geographical Information Systems: principles and applications, London: Longman, Vol. 1, pp. 9-20
MITCHELL, W. L. (1995) City of Bits, Cambridge (Mass.)/ London (UK): MIT-Press, $225 \mathrm{pp}$.

NCGIA (1989): The Research Plan of the National Center for Geographic Information and Analysis, International Journal of Geographical Informations Systems, Vol. 3, No. 2, pp. 117-136

NCGIA (1995): Advancing Geographic Information Science; A proposal to NSF, Project description. http://www.ncgia. ucsb.edu/secure/secC.html\#RTFToC12

NATIONAL ACADEMY OF SCIENCE (NAS) - National Research Council (NRC) (1965): The Science of Geography, Washington: NAS/NRC

SCHAEFER, F.K. (1953): Exceptionalism in geography: a methodological examination, Annals AAG, Vol. 43, pp. 226-249

SCHMIDT, A.H., and ZAFFT, W.D. (1975): Programs of the Harvard University Laboratory for Computer Graphics and Spatial Analysis. In: Davis, J.C., and MacCullagh, M.C. (1975): Display and Analysis of Spatial Data, London: Wiley SCHULZ, H.-D. (1980): Die deutschsprachige Geographie von 1800 bis 1970. Ein Beitrag zur Geschichte ihrer Methodologie, Abhandlungen des Geographischen Instituts der FU Berlin, Vol. 29, 478 S.

STARR, L. E., and ANDERSON, K. E. (1991): A USGS Perspective on GIS, in: Maguire, D. J., Goodchild, M. F., Rhind D. W., (eds.) Geographical Information Systems: principles and applications, London: Longman, Vol. 2, pp. 11-22

TOMLINSON, R. F. (1967): An Introduction to the Geographic Information System of the Canada Land Inventory, Ottawa: Dept. of Forestry and Rural Development

TOMLINSON, R. F. (ed.) (1972): Geographic Data Handling, Ottawa: Commission on Geographical Data Sensing and Processing, International Geographical Union VCKOVSKI, A. (1998): Interoperable and Distributed Processing in GIS, London: Taylor \& Francis 